\title{
Efficient DTN Routing Protocol
}

\author{
Mamoun Hussein Mamoun \\ Faculty of Computer Science and Information \\ Al Jouf University \\ Al Jouf, Saudi Arabia
}

\begin{abstract}
This paper presents a novel routing protocol for Disruption Tolerant Networks (DTNs) called Efficient DTN Routing Protocol $(E D R P)$. EDRP smartly integrates the forwarding and buffer management policies into an adaptive protocol that includes a local network parameters estimation mechanism. It dynamically adjusts the delivery probability for messages according to a new metric. Meanwhile, EDRP arranges the forwarding sequence and the dropping priority based on their assigned weight. The weight is determined by the Replication Density $(R D)$, the Message Length $(M L)$, and Message Remaining Life Time (MRLT). An extensive simulation of $E D R P$ was carried out and its performance was compared to well known $D T N$ routing protocols: PRoPHET, and Epidemic Routing protocols. Simulation results show that the proposed routing protocol outperforms them in terms of packet delivery ratio, delivery delay and message overhead.
\end{abstract}

\section{General Terms}

DTN networks, Opportunistic network, Adaptive routing protocols, Forwarding and Dropping, New routing protocols.

\section{Keywords}

DTN, Adaptive Routing, Weight Estimation, Drop Policy.

\section{INTRODUCTION}

$D T N$ networks are one of the most interesting evolutions of classic Mobile Ad Hoc Networks (MANET). The main assumption of MANET environments is that a sender and a destination are connected to the network at the same time. If the destination is not connected when the sender wishes to transmit messages, they get dropped at some point of the network. However, in a pervasive networking environment, nodes will be seldom connectable at the same time through a multi-hop path. For example, devices that users carry with them might be only sporadically attached to the Internet, e.g. when the user moves close to an Access Point. In other words, it is foreseeable a scenario in which a large number of wireless devices and limited-size networks will be just occasionally connected to each other. DTN networks aim at make users able to exchange data even in such a disconnected environment, by opportunistically exploiting any nearby device to move messages closer to the final destination. To this end, legacy protocols designed for MANET should be drastically redesigned [1], [2], [3]. Currently, envisioning routing and forwarding protocols 1 for DTN networks is one of the most exciting topics [4]. In opportunistic networks, the traditional routing paradigm of Internet and MANET, in which routes are computed based exclusively on topological information, is not adequate anymore. A first approach to routing in DTN networks is some variation of controlled flooding: Messages are flooded with limited Time-To-Live $(T T L)$, and delivered to the destination as soon as it gets in touch with some node that received the message during the flood [5]. Many researchers have proposed new routing protocols such as Epidemic [5], Prophet [6], Spray-andWait[7], Spray-and-Focus [8], MaxProp [9] , ORWAR [10], ERS [11], APRP [12], and PFBR [13] to handle this specific problem for DTN.

In this paper, an Efficient Routing Protocol (EDRP) for DTNs was proposed. EDRP smartly integrates the forwarding and buffer management policies into an adaptive protocol that includes a local network parameters estimation mechanism It arranges the forwarding sequence and the dropping priority based on their assigned weight. The weight is determined by the three local parameters, namely, Replication Density $(R D)$, Message Length $(M L)$, and Message Remaining Life Time (MRLT).

The remainder of this paper is organized as follows: Section 2 presents the related work. In section 3 our proposed mechanism is described. Simulation and results of our proposed routing protocol can be found in section 4. Section 5 discusses conclusion.

\section{Related Work}

In the past few years, many routing algorithms are proposed in DTN network, such as Epidemic routing, Spray and wait, PROPHET, and so on. The basic idea of them is to increase identical copies of data into network and rely on node mobility to transmit the copies toward the destination. Obviously if there are more copies in network, the better delay performance tends to achieved in opportunistic network. But its drawback is that the traffic overhead is tremendous. If network resources are limited, replication based schemes will degrade the network performance. The reader can find a comprehensive survey on routing protocol for DTN network.

\subsection{Epidemic}

The Epidemic routing [5] protocol is a flooding-based scheme. Each node has two buffers, the first one for storing the messages generated by itself, and the second one for those messages received from other nodes. Each message is tagged with a unique ID. Each node also maintains a list of message IDs that it is currently holding in its buffer called the Summary Vector. When two nodes meet, they exchange their Summary Vectors with each other. By comparing these Summary Vectors, the nodes exchange those messages which they do not have with them. When this operation of message exchange is completed, all nodes have the same messages in their buffers. This creates a large amount of redundancy in the network, which incurs significant demand on both bandwidth and buffer capacity, but at the same time, makes it extremely robust to node and network failure. The simulation results obtained in this work show that for this protocol, the message delivery ratio is very high and the message is delivered in minimum amount of time if sufficient resources are available. 


\subsection{PRoPHET}

In PRoPHET (Probabilistic Routing Protocol using History of Encounters and Transitivity) [6], before sending a message, each node estimates a probabilistic metric called Delivery Predictability for each known destination. It indicates the probability of successful delivery of a message to the destination from the source node. The calculation of the Delivery Predictability is based on the history of encounters between nodes or history of visits to certain locations. When two nodes meet, they exchange their Summary Vectors containing the Delivery Predictability. If two nodes are often encountered, they have high Delivery Predictability to each other. On the other hand, if a pair of nodes does not find each other for a period of time, they are not good forwarders of the message to each other. Hence, the Delivery Predictability value must decrease with time. Thus, a message is forwarded to a node from a set of available nodes which has the higher value of Delivery Predictability among them to the destination node. The simulation results obtained in this work show that this protocol has less message exchanges, less communication overhead, less delay, and higher delivery success rate as compared to the Epidemic Routing.

\subsection{Spray and Wait}

This protocol [7] provides an interesting technique to control the level of flooding. The message is mainly delivered in two phases: the Spray phase and the Wait phase. For every message originating at the source node, L copies of the message are spread over the network by the source node and other nodes receive a copy of the message from the source node to L distinct relays. In the Wait phase if the destination was not found during the spray phase, each relay node having a copy of the message performs the direct transmission. The simulation results show that this protocol has less number of transmissions and less delivery delay as compared to the Epidemic Routing.

\section{PROPOSED RPUTING PROTOCOL}

\subsection{Adaptive Forwarding Algorithm}

Suppose that node a has a message $\mathrm{m}$ for the destination $\mathrm{d}$. When a contact occurs between a pair of nodes a and $b$, node $a$ forwards the message $m$ to node $b$ only if $b$ has a greater Delivery Predictability to the destination d. The delivery predictability is given by [6]

$$
\begin{gathered}
P_{(a b)}=\left\{\begin{array}{c}
P_{(a b) \text { old }}+\left(1-P_{(a b) \text { old }}\right) * P_{\text {init }}, m_{a b}=0 \\
P_{(a b) \text { old }} * \gamma^{k}, m_{a b}=1
\end{array}\right. \\
\text { Where }\left\{\begin{array}{c}
\gamma=0.98 \\
k=\frac{T_{\text {now }}-T_{\text {lastmeet }}}{\text { AgingInterval }} \\
P_{\text {init }}=0.75
\end{array}\right.
\end{gathered}
$$

This formula just reflects the historical probability. However, if a node has a very high probability without any residual energy, it would not be a perfect potential relay due to that the node may exhaust its power before it hand over the message to next node closer to the destination. A new parameter $\boldsymbol{E}_{c}$ is inducted to reflect the surplus power of the current node. Then the power influent formula is given by

$$
P_{(a b)}^{\prime}=P_{(a b)} \xi+(1-\xi) E_{c}
$$

where $\xi$ is a variable which weight the influence about the encountering probability and the remnant energy. In proposed routing, we have chosen a rather simple forwarding strategywhen two nodes meet, a message to be transferred to other node if the delivery predictability $P^{\prime}{ }_{(a, b)}$ of the destination of the message is higher at the other destination nodes.

\subsection{Priority Mechanism}

Challenged networks are characterized by short contact time between the nodes, resulting from high speeds or high contention. In order to appropriately prioritize messages for dropping and forwarding, EDRP employs three metrics to calculate the priority for each message: replications density $(R D)$, message length $(M L)$, and message remaining life time (MRLT). The rationale behind this prioritization is twofold:

- Messages which propagated enough in the network should have less priority in order to give a chance for less disseminated messages. Message dissemination is measured by Replications Density $(R D) . R D$ is calculated as follows: Let $M_{i}\left(T_{s}\right)$ represents the number of message i copies on encounters and $N\left(T_{s}\right)$ represents the total number of nodes that the node encounters in time interval $T_{s}$. Then, the $R D$ of message $\mathrm{i}$ is given by

$$
R D\left(T_{s}\right)=\frac{M\left(T_{s}\right)}{N\left(T_{s}\right)}
$$

In the special case, when a node is isolated during the period, $T_{t h}$ seconds, the value of RD will be remain until a new node is encountered and the process of calculated the RD is started.

- Large messages should have less priority in order to maximize the number of messages that can be successfully transmitted in each short contact opportunity. It is argued in [14] that this approach reduces partial transmissions and increases the number of packets delivered.

- Forwarding messages with longer remaining life time $(M R L T)$ are scheduled to be sent first because they have a higher probability to reach its destination. then the sequence of delivering messages is very important.

The priority of message $i$ in $j^{\text {th }}$ sample duration can be calculated as

$$
\operatorname{priorty}(i)_{j}=C_{1} \times R D(i)_{j}+C_{2} \times M L_{i}+C_{3} \times \operatorname{MRLT}(i)_{j}
$$

where $C_{1}, C_{2}$, and $C_{3}$ are priorities of the metrics.

Calculating the values of metric priorities is a typical problem of Multiple Attribute Decision Making (MADM). In this paper, we assume that $R D$ is the most important metric and $M R L T$ is the least important and using the Three Standard Degree Method of Analytic Hierarchy Process [15] in order to determine $C_{1}, C_{2}$, and $C_{3}$. 


\subsection{ACK Vector Exchange Scheme}

Since messages are forwarded by asynchronous transmission mode in DTN, most of the intermediate nodes of a message can hardly know whether a copy of this message has been delivered to the destination. In this case, even if a message has been successfully delivered, there still exist multiple copies of the message in the network consuming the resources unnecessarily. Therefore, we adopt an ACK vector exchange mechanism that is similar to the analysis in [16] to reduce such overhead.

\section{PERFORMANCE EVALUATION}

This section presents a comparative simulation analysis of EDRP with PRoPHET, and Epidemic routing protocols. We have implemented a DTN framework into One Simulator [17] to evaluate our protocol. When evaluating the performance of the proposed protocol, it is very important that the mobility models employed are representative and realistic. Thus, DTN network used for evaluation consists of two groups of pedestrians (15 elements each), a group of cars (15 elements) and three groups of trains with two nodes (two nodes in each train). The sum of all groups equals 51 nodes. The used scenario corresponds to the map of the city of Helsinki, in Finland, which includes streets and crosswalks. All nodes were considered with the same equipment type, although in the case of trains that option is a bit forced. The equipment used is a mobile phone with Bluetooth interface, communication range of 10 meters, transmission speed of $250 \mathrm{Kbps}$ and storage capacity of $5 \mathrm{MB}$. This phone has a battery capacity of 4,8 joules.

Each pedestrian group uses the model Shortest Path MapBased Movement. In this model, the node walks always in the available map roads by choosing a random point and using the shortest route to this destination from its current location. The used speed can vary between 0.5 to 1.5 meters per second. Cars are forced to follow only the roads, traveling at a speed between 10 and $50 \mathrm{~km} / \mathrm{h}$. They also follow the same pattern of movement of pedestrians. For simulation purposes, it is assumed that each car is driven by a person who has the same mobile phone model of the pedestrians. The three groups of trains use the Routed Map-Based Movement model. In this model, each node moves always through the same route. Each group has a different route and stops between ten to thirty seconds at certain locations on the map. Their velocities vary between 25 and $36 \mathrm{~km} / \mathrm{h}$. As the simulator have the limitation that people cannot leave or enter the train, it was assumed that train drivers have the same mobile phone model of the other groups.

Furthermore, all nodes use the same equipment similar to a Smart phone with Bluetooth interface. The scenario used provided with very heterogeneous groups of nodes with different speeds and movements. Other parameters for the simulation are presented in the following table.(Table 1)
Table 1. Simulation Parameters

\begin{tabular}{|c|c|}
\hline Parameter & Value \\
\hline No. of Nodes & 51 \\
\hline Simulation Time & 14 days \\
\hline Generation Message Rate & $25-30 \mathrm{sec}$ \\
\hline Size of Messages & $0.5-1 \mathrm{MB}$ \\
\hline Battery Capacity & 4800 \\
\hline ScanEnergy & $0.92 \mathrm{~W} / \mathrm{sec}$ \\
\hline TransmitEnergy & $0.08 \mathrm{~W} / \mathrm{sec}$ \\
\hline ReceiveEnergy & $0.08 \mathrm{~W} / \mathrm{sec}$ \\
\hline
\end{tabular}

Fig. 1 shows the delivery ratio as the buffer size is varied, for PRoPHET Routing, Epidemic Routing, and EDRP. The delivery ratios of all routing protocols increase as the buffer size increases. Among these DTN routing protocols, the EDRP achieves the highest delivery ratio. This is because the EDRP has better mechanism for message priority mechanism to determine their priority for forwarding and for dropping. However, as the buffer size increase the buffer constraint is removed and other protocols start to deliver more messages.

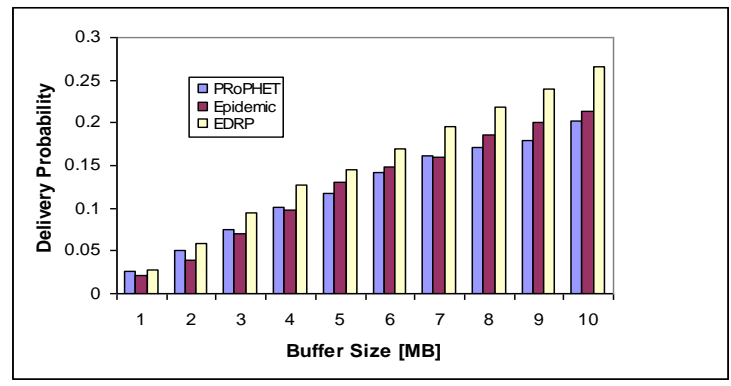

Fig 1: Delivery probability as function of buffer size

Fig. 2 shows the average delay of a message as the buffer size varies. Similar to the delivery ratio, the result shows that the performance of EDRP is better than those of PRoPHET, and Epidemic. The reason for the shorter average delay is because messages are prioritized and the network is not so congested as $E D R P$ uses an adaptive forwarding algorithm to minimize network congestion and messages are prioritized for forwarding by a node. The simulation results show that EDRP achieves a $6.7 \%$ reduction in average delivery delay over PRoPHET, and Epidemic routing protocols.

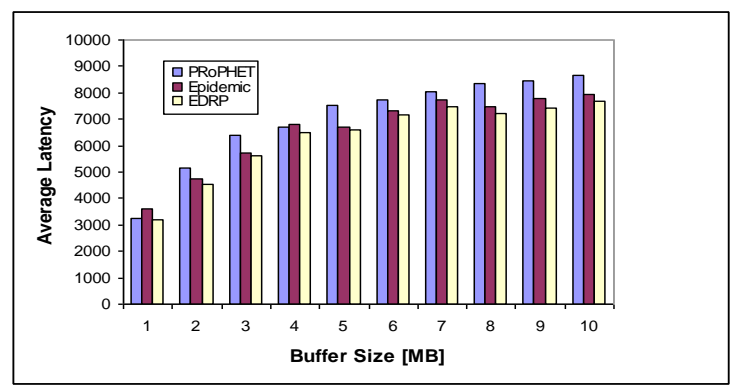

Fig 2: Average latency as function of buffer size 
Fig.3. EDRP utilizes the ACK vector exchange mechanism to purge the redundant messages, as the simulation results indicate, the copies of each message are much less than PRoPHET and Epidemic Routing protocols.

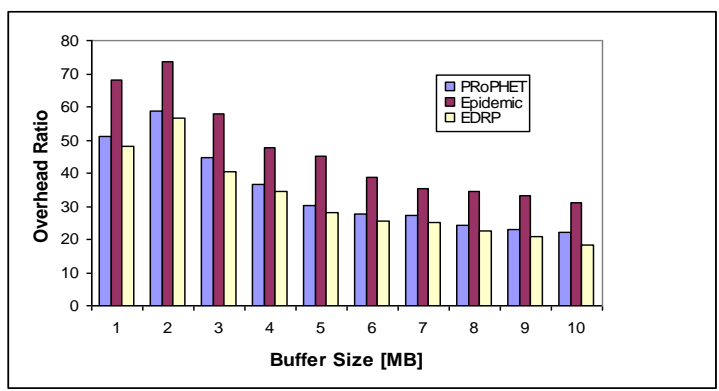

Fig 3: Overhead ratio as function of buffer size

\section{CONCLUSION}

This paper introduces an efficient message forwarding mechanism in Disruption Tolerant Networks. A novel and simple scheme, called EDRP, which managed to address some of the shortcomings of $D T N$ routings in limited buffer or bandwidth network environment is proposed. EDRP employed an adaptive probability to calculate message priority for message forwarding. Meanwhile, EDRP adopted acknowledge mechanism to purge successfully delivered message in source and intermediate nodes. Simulation results have shown that EDRP outperforms PRoPHET Routing, and Epidemic Routing by a significant margin.

\section{REFERENCES}

[1] K. Fall, "A delay-tolerant network architecture for challenged internets", Proc. of ACM SIGCOMM, pp 2734, Aug., 2003

[2] S. Jain, K. Fall, and R. Patra, "Routing in a delaytolerant network", Proc. of ACM SIGCOMM, pp 145158, 2004.

[3] J. Scott, P. Hui, J. Crowcroft, C. Diot, "Haggle: A Networking Architecture Designed Around Mobile Users" Proc. $3^{\text {rd }}$ Annual Conference of IFIP WONS, Jan. 2006.

[4] L. Pelusi, A. Passarella, and M. Conti, "Opportunistic Networking: data forwarding in disconnected mobile ad hoc networks", IEEE Communications Magazine, vol. 44, no. 11, pp. 134- 141, Nov. 2006.

[5] A. Vahdat and D. Becker, "Epidemic routing for partially connected ad hoc networks", Tech. Rep. CS2000-06, CS Dept., Duke University, April 2000.
[6] A. Lindgren et al, "Probabilistic Routing in Intermittently Connected Networks", Mobile Comp. and Comm. Rev, vol. 7, no. 3, pp. 19- 20, July 2003.

[7] T. Spyropoulos, K. Psounis, and C. S. Raghavendra, "Spray and wait: Efficient routing in intermittently connected mobile networks", In Proceedings of ACMSIGCOMM workshop on Delay Tolerant Networking (WDTN'5), pp 252-259, 2005.

[8] T. Spyropoulos, K. Psounis, and Cauligi S. Raghavendra, "Spray and Focus Efficient Mobility-Assisted Routing for Heterogeneous and correlated Mobility", in Proc. PerCom. Workshops apos, pp 79-85, March 2007.

[9] J. Burgess, B. Gallagher, D. Jensen, and B. N. Levine, "MaxProp: Routing for Vehicle-Based DisruptionTolerant Networks", In Proc. IEEE Infocom, pp. 1-11, IEEE, April 2006.

[10] Gabriel Sandulescu, and Simin Nadjm-Tehrani, "Opportunistic DTN routing with window-aware adaptive replication", AINTEC' 08, November, 18- 20, Bangkok, Thailand.

[11] Mamoun H. M., "Efficient Routing Scheme for Opportunistic Networks ", International Journal of Engineering and Technology, Vol. 2, No 6, pp. 940-945, June 2012.

[12] Mamoun H. M., S. El Barrak "Adaptive Priority Routing Protocol for DTN Networks ", International Journal of Engineering and Technology, Vol. 3, No 3, pp. 258-264, March 2013.

[13] Mamoun H. M. and, S. El Barrak, "Proposed Fuzzy Based Routing for DTN", International Journal of Computer Applications, Vol. 66, No 6, pp. 19-22, March 2013.

[14] A. Mathurapoj, C. Pronavalai and G. Chakraborty, "Fuzzy-spray: Efficient routing in delay tolerant ad-hoc network based on fuzzy decision mechanism", Fuzzy Systems, 2009. FUZZ-IEEE 2009. IEEE International Conference on, IEEE, 2009, PP. 104-109.

[15] Shubo Xu, "Theory of Analytic and Hierarchy Process", Tianjin University, 1988.

[16] X. Zhang, G. Kurose, and D. Twosley, "Performance modeling of epidemic routing", in Proceeding of IFIP Networking, 2006.

[17] Ari Keronen, and Jurg Ott, "The One simulator for DTN protocol evaluation", in SIMUTools 2009, Rom, Italy. 